\title{
Recent Developments on Optimized Schwarz Methods
}

\author{
Nataf Frédéric ${ }^{1}$ \\ CMAP, CNRS UMR 7641, Ecole Polytechnique, 91128 Palaiseau Cedex \\ nataf@cmap.polytechnique.fr
}

\section{Introduction}

The classical Schwarz method [31] is based on Dirichlet boundary conditions. Overlapping subdomains are necessary to ensure convergence. As a result, when overlap is small, typically one mesh size, convergence of the algorithm is slow. A first possible remedy is the introduction of Neumann boundary conditions in the coupling between the local solutions. This idea has lead to the development of the Dirichlet-Neuman algorithm [10], Neumann-Neumann method [3] and FETI methods [8]. These methods are widely used and have been the subject of many works, improvements and extensions to various scalar or systems of partial differential equations, see for instance the following books [32], [27], [37] and [35] and references therein. A second cure to the slowness of the original Schwarz method is to use more general interface conditions, Robin conditions were proposed in [19] and pseudo-differential ones in [17]. These methods are well-suited for indefinite problems [5] and as we shall see to heterogeneous problems.

We first recall the basis for the optimized Schwarz methods in $\S 2$ and an application to the Helmholtz problem in $\S 2.2$. Then, we consider equations with highly discontinuous coefficients in $\S 3$. We present an optimized Schwarz method that takes into account properly the discontinuities and make comparisons with other domain decomposition methods.

\section{Generalities on Optimized Schwarz methods}

\subsection{Optimal Interface Conditions}

We show exhibit interface conditions which are optimal in terms of iteration counts. The corresponding interface conditions are pseudo-differential and are not practical. Nevertheless, this result is a guide for the choice of partial differential interface conditions. Moreover, this result establishes a link between 
the optimal interface conditions and artificial boundary conditions. This is also a help when dealing with the design of interface conditions since it gives the possibility to use the numerous papers and books published on the subject of artificial boundary conditions, see e.g. [6, 15].

We consider a general linear second order elliptic partial differential operator $\mathcal{L}$ and the problem:

Find $u$ such that $\mathcal{L}(u)=f$ in a domain $\Omega$ and $u=0$ on $\partial \Omega$.

The domain $\Omega$ is decomposed into two subdomains $\Omega_{1}$ and $\Omega_{2}$. We suppose that the problem is regular so that $u_{i}:=\left.u\right|_{\Omega_{i}}, i=1,2$, is continuous and has continuous normal derivatives across the interface $\Gamma_{i}=\partial \Omega_{i} \cap \bar{\Omega}_{j}, i \neq j$.

Fig. 1. A two-subdomain decomposition

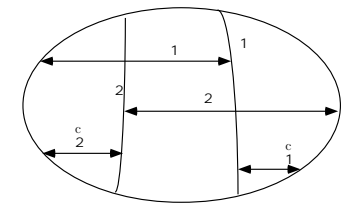

A generalized Schwarz type method is considered.

$$
\begin{array}{ll}
\mathcal{L}\left(u_{1}^{n+1}\right)=f \quad \text { in } \Omega_{1} & \mathcal{L}\left(u_{2}^{n+1}\right)=f \quad \text { in } \Omega_{2} \\
u_{1}^{n+1}=0 \text { on } \partial \Omega_{1} \cap \partial \Omega & u_{2}^{n+1}=0 \text { on } \partial \Omega_{2} \cap \partial \Omega \\
\mu_{1} \nabla u_{1}^{n+1} \cdot \mathbf{n}_{1}+\mathcal{B}_{1}\left(u_{1}^{n+1}\right) & \mu_{2} \nabla u_{2}^{n+1} \cdot \mathbf{n}_{\mathbf{2}}+\mathcal{B}_{2}\left(u_{2}^{n+1}\right) \\
=-\mu_{1} \nabla u_{2}^{n} \cdot \mathbf{n}_{\mathbf{2}}+\mathcal{B}_{1}\left(u_{2}^{n}\right) \text { on } & \Gamma_{1}=-\mu_{2} \nabla u_{1}^{n} \cdot \mathbf{n}_{1}+\mathcal{B}_{2}\left(u_{1}^{n}\right) \text { on } \Gamma_{2}
\end{array}
$$

where $\mu_{1}$ and $\mu_{2}$ are real-valued functions and $\mathcal{B}_{1}$ and $\mathcal{B}_{2}$ are operators acting along the interfaces $\Gamma_{1}$ and $\Gamma_{2}$. For instance, $\mu_{1}=\mu_{2}=0$ and $\mathcal{B}_{1}=\mathcal{B}_{2}=\mathrm{Id}$ correspond to the original Schwarz method; $\mu_{1}=\mu_{2}=1$ and $\mathcal{B}_{i}=\alpha \in \mathbf{R}$, $i=1,2$, has been proposed in [19] by P. L. Lions.

The question is:

Are there other possibilities in order to have convergence in a minimal number of steps?

In order to answer this question, we introduce the DtN (Dirichlet to Neumann) map (a.k.a. Steklov-Poincaré) of domain $\Omega_{2} \backslash \bar{\Omega}_{1}$ : Let

$$
\begin{gathered}
u_{0}: \Gamma_{1} \rightarrow \mathbf{R} \\
\operatorname{DtN}_{2}\left(u_{0}\right):=\nabla v \cdot n_{2 \mid \partial \Omega_{1} \cap \bar{\Omega}_{2},}
\end{gathered}
$$

where $n_{2}$ is the outward normal to $\Omega_{2} \backslash \bar{\Omega}_{1}$, and $v$ satisfies the following boundary value problem:

$$
\begin{array}{rlrl}
\mathcal{L}(v) & =0 \quad \text { in } \quad & \Omega_{2} \backslash \bar{\Omega}_{1} \\
v & =0 \quad \text { on } & \partial \Omega_{2} \cap \partial \Omega \\
v & =u_{0} & \text { on } & \partial \Omega_{1} \cap \bar{\Omega}_{2} .
\end{array}
$$


Similarly, we can define $D t N_{1}$ the Dirichlet to Neumann map of domain $\Omega_{1} \backslash$ $\bar{\Omega}_{2}$. The following optimality result is proved in [23]:

Result 1 The use of $\mathcal{B}_{i}=\operatorname{DtN}_{j}(i=1,2$ and $i \neq j)$ as interface conditions in (1) is optimal: we have (exact) convergence in two iterations.

The two-domain case for an operator with constant coefficients has been first treated in [17]. The multidomain case for a variable coefficient operator with both positive results [25] and negative conjectures [26] has been considered as well.

Remark 1. The main feature of this result is to be very general since it does not depend on the exact form of the operator $\mathcal{L}$ and can be extended to systems or to coupled systems of equations as well with a proper care of the well posedness of the algorithm.

As an application, we take $\Omega=\mathbf{R}^{2}$ and $\left.\Omega_{1}=\right]-\infty, 0[\times \mathbf{R}$. Using the Fourier transform along the interface (the dual variable is denoted by $k$ ), it is possible to give the explicit form of the $\mathrm{DtN}$ operator for a constant coefficient operator. If $\mathcal{L}=\eta-\Delta$, the $\mathrm{DtN}$ map is a pseudo-differential operator whose symbol is

$$
B_{i, \text { opt }}(k)=\sqrt{\eta+k^{2}},
$$

i.e., $\mathcal{B}_{i, \text { opt }}(u)(0, y)=\int_{\mathbf{R}} B_{i, \text { opt }}(k) \hat{u}(0, k) e^{I k y} d k$.

The symbol is not polynomial in the Fourier variable $k$ so that the operators and hence the optimal interface conditions are not a partial differential operator. They correspond to exact absorbing conditions. These conditions are used on the artificial boundary resulting from the truncation of a computational domain. On this boundary, boundary conditions have to be imposed. The solution on the truncated domain depends on the choice of this artificial condition. We say that it is an exact absorbing boundary condition if the solution computed on the truncated domain is the restriction of the solution of the original problem. Surprisingly enough, the notions of exact absorbing conditions for domain truncation and that of optimal interface conditions in domain decomposition methods coincide.

\subsection{Optimized Interface Conditions for the Helmholtz equation}

As the above example shows, the optimal interface conditions are pseudodifferential. Therefore they are difficult to implement. Moreover, in the general case of a variable coefficient operator and/or a curved boundary, the exact form of these operators is not known, although they can be approximated by partial differential operators which are easier to implement. The approximation of the DtN has been addressed by many authors since the seminal paper [6] by Engquist and Majda on this question. A first natural idea is to use these works in domain decomposition methods. As we shall see, it is better to 
design approximations that are optimized with respect to the domain decomposition method. We seek approximations to the Dirichlet to Neumann map by a partial differential operator

$$
D t N \simeq \alpha_{o p t}-\frac{\partial}{\partial \tau}\left(\gamma_{o p t} \frac{\partial}{\partial \tau}\right)
$$

where $\partial_{\tau}$ is the derivative along the interface. The parameters are chosen in order to minimize the convergence rate of the algorithm. These interface conditions are called optimized of order 2 conditions (opt2). If we take $\gamma=0$, the optimization is performed only w.r.t. $\alpha$, they are called optimized of order 0 (opt0). The idea was first raised in [34]. But the link with the optimal interface conditions was not done and made the optimization too complex.

As an example, we present here the case of the Helmholtz equation that was considered in [12]. We want to solve by a domain decomposition method:

$$
\mathcal{L}(u)=\left(-\omega^{2}-\Delta\right)(u)=f
$$

In order to find the optimized interface conditions, we first consider a very simple geometry for which the optimization is tractable and then apply these results to an industrial case. As a first step, the domain $\Omega=\mathbb{R}^{2}$ is decomposed into two non overlapping subdomains $\Omega_{1}=(-\infty, 0) \times \mathbb{R}$ and $\Omega_{2}=(0, \infty) \times \mathbb{R}$. The algorithm is defined by (1) with $\mu_{1}=\mu_{2}=1$ and $\mathcal{B}_{1}=\mathcal{B}_{2}=\alpha-\frac{\partial}{\partial \tau}\left(\gamma \frac{\partial}{\partial \tau}\right)$. A direct computation yields the convergence rate of the iterative method in the Fourier space:

$$
\rho(k ; \alpha, \gamma) \equiv\left\{\begin{array}{l}
\left|\frac{I \sqrt{\omega^{2}-k^{2}}-\left(\alpha+\gamma k^{2}\right)}{I \sqrt{\omega^{2}-k^{2}}+\left(\alpha+\gamma k^{2}\right)}\right| \text { if }|k|<\omega \quad\left(I^{2}=-1\right) \\
\left|\frac{\sqrt{k^{2}-\omega^{2}}-\left(\alpha+\gamma k^{2}\right)}{\sqrt{k^{2}-\omega^{2}}+\left(\alpha+\gamma k^{2}\right)}\right| \text { if } \omega<|k|
\end{array}\right.
$$

The convergence rate in the physical space is the maximum over $k$ of $\rho(k ; \alpha, \gamma)$. Actually, it is sufficient to consider Fourier modes that can be represented on the mesh used in the discretization of the operator. It imposes a truncation in the frequencey domain of the type $|k|<\pi / h$ where $h$ is the mesh size. We have then to minimize the convergence rate in the physical space with respect to the parameters $\alpha$ and $\gamma$. We are thus led to the following min-max problem:

$$
\min _{\alpha, \gamma} \max _{|k|<\pi / h} \rho(k ; \alpha, \gamma)
$$

Under additional simplifications, we get analytic formulas for the optimized parameters $\alpha$ and $\gamma$ depending on $\omega$ and $h$, see [12]. 
For an arbitrary domain decomposition for instance obtained by an automatic mesh partitioner as the one shown on figure 2, we proceed in the following manner. At each node on the interface, we use the local value of the mesh size to compute the optimized parameters using the formula established in the simple case of the plane $\mathbb{R}^{2}$ divided into two half-planes. In table 1, we give iteration counts for various interface conditions: $\mathrm{ABC} 0$ means that the interface conditions are $\partial_{n}+I \omega$ (i.e. $\alpha=I \omega$ and $\gamma=0$, see [2]), $\mathrm{ABC} 2$ corresponds to absorbing conditions of order 2 that are currently used for truncation of domains see [6] but were not designed with domain decomposition methods in mind. Notice that since the interfaces are not straight lines and the subdomains have an irregular shape, we are very far from the ideal case considered above. Nevertheless, the optimized interface conditions perform quite well.

Fig. 2. Domain decomposition of the cabin car

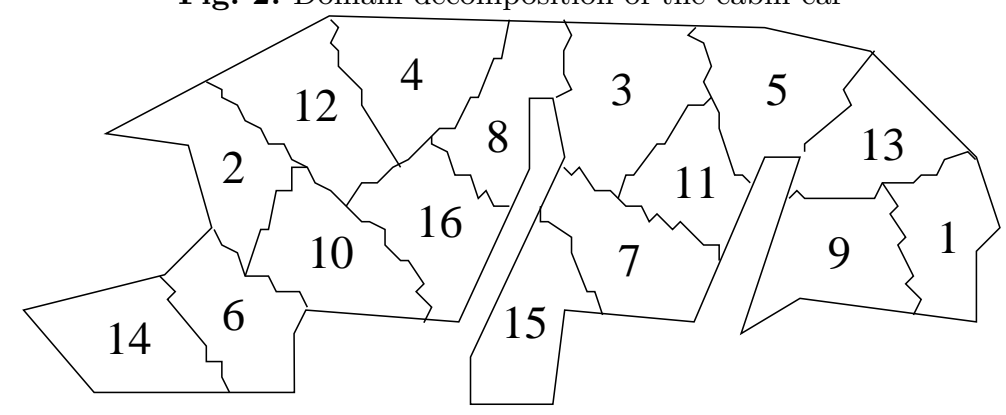

Table 1. Iteration Counts for various interface conditions and numbers of subdomains $N_{s}$

\begin{tabular}{rrrrr}
\hline$N_{s}$ & ABC 0 & ABC 2 & Optimized \\
\hline 2 & 16 it & 16 it & 9 it \\
4 & 50 it & 52 it & 15 it \\
8 & 83 it & 93 it & 25 it \\
16 & 105 it & 133 it & 34 it \\
\hline
\end{tabular}

\section{Optimized Schwarz Method for Highly Discontinuous Coefficients}

We consider now a symmetric positive definite problem but with highy discontinuous coefficients. The model equation is 


$$
\eta u-\operatorname{div}(\kappa \nabla u)=f \text { in } \Omega
$$

It contains some of the difficulties typical of porous media flow simulations. Indeed, the coefficients $\eta$ and $\kappa$ have jumps which are typically of four orders of magnitude. The tensor $\kappa$ is anisotropic with large anisotropy ratios: $10^{-4} \leq$ $\kappa_{x} / \kappa_{y} \leq 10^{4}$. In the situation we consider, the domain $\Omega$ is divided into two subdomains $\Omega_{1}$ and $\Omega_{2}$ corresponding to two different geological blocks. Each subdomain is layered so that the coefficients are discontinuous both across and along the interface, see for instance figure 3. These kinds of problems lead to very ill-conditioned linear systems so that there are plateaus in the convergence of Krylov methods even with otherwise "good" preconditioners.

Fig. 3. Lithology

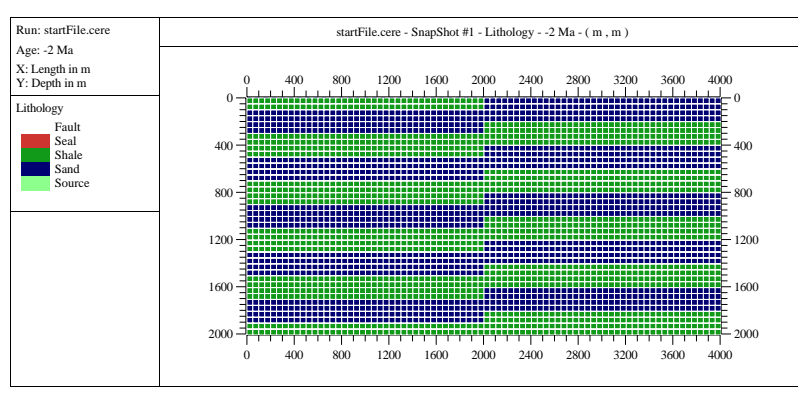

In order to design optimized interface conditions, we first define more precisely the model problem we consider.

\subsection{Setting of the semi-discrete problem}

We consider a model problem set in an infinite tube $\Omega=\mathbb{R} \times \omega$ where $\omega$ is some bounded open set of $\mathbb{R}^{p}$ for some $p \geq 1$. The domain is decomposed into two non overlapping half tubes $\Omega_{1}=(-\infty, 0) \times \omega$ and $\Omega_{2}=(0, \infty) \times \omega$. A point in $\Omega$ will be denoted by $(x, \mathbf{y})$. Let for $i=1,2$

$$
\mathcal{L}_{i}:=-\frac{\partial}{\partial x} c_{i}(\mathbf{y}) \frac{\partial}{\partial x}+\mathcal{C}_{i}(\mathbf{y})
$$

where $c_{i}$ is a positive real valued function and $\mathcal{C}_{i}$ is a symmetric positive definite operator independent of the variable $x$. For instance, if $p=2$ one might think of

$$
\mathcal{C}_{i}:=\eta_{i}(y, z)-\left(\frac{\partial}{\partial y} \kappa_{i, y}(y, z) \frac{\partial}{\partial y}+\frac{\partial}{\partial z} \kappa_{i, z}(y, z) \frac{\partial}{\partial z}\right)
$$

with homogeneous Dirichlet boundary conditions and $\eta_{i} \geq 0, c_{i}, \kappa_{i, y}, \kappa_{i, z}>0$ are given real-valued functions and $(y, z) \in \omega$.

We want to solve by a domain decomposition method the following problem 


$$
\begin{aligned}
& \mathcal{L}_{i}\left(u_{i}\right)=f \text { in } \Omega \\
& u=0 \text { on } \partial \Omega
\end{aligned}
$$

with

$$
C_{1} \frac{\partial u_{1}}{\partial x}=C_{2} \frac{\partial u_{2}}{\partial x} \quad \text { on } \quad \Gamma
$$

and

$$
u_{2}=u_{1} \quad \text { on } \quad \Gamma
$$

The problem can be considered at the continuous level and then discretized (see e.g. [12], [11], [24] ), or at the discrete level (see e.g. [20], [28] or [13]). We choose here a semi-discrete approach where only the tangential directions to the interface $x=0$ are discretized whereas the normal direction $x$ is kept continuous.

We therefore consider a discretization in the tangential directions which leads to

$$
\mathcal{L}_{i, h}:=-\frac{\partial}{\partial x} C_{i} \frac{\partial}{\partial x}+B_{i}
$$

where $B_{i}$ and $C_{i}$ are symmetric positive matrices of order $n$ where $n$ is the number of discretization points of the open set $\omega \subset \mathbb{R}^{p}$. For instance if we take $\mathcal{C}_{i}$ to be defined as in (4), $B_{i}$ may be obtained via a finite volume or finite element discretization of (4) on a given mesh or triangulation of $\omega \subset \mathbb{R}^{2}$.

We consider a domain decomposition method based on arbitrary interface conditions $\mathcal{D}_{1}$ and $\mathcal{D}_{2}$. The corresponding Optimized Schwarz method (OSM) reads:

$$
\begin{aligned}
& \mathcal{L}_{1, h}\left(u_{1}^{n+1}\right)=f \quad \text { in } \quad \Omega_{1} \quad \mathcal{L}_{2, h}\left(u_{2}^{n+1}\right)=f \quad \text { in } \quad \Omega_{2} \\
& \mathcal{D}_{1}\left(u_{1}^{n+1}\right)=\mathcal{D}_{1}\left(u_{2}^{n}\right) \quad \text { on } \quad \Gamma \quad \mathcal{D}_{2}\left(u_{2}^{n+1}\right)=\mathcal{D}_{2}\left(u_{1}^{n}\right) \quad \text { on } \quad \Gamma
\end{aligned}
$$

where $\Gamma$ is the interface $x=0$. It is possible to both increase the robustness of the method and its convergence speed by replacing the above fixed point iterative solver by a Krylov type method. This is made possible by substructuring the algorithm in terms of interface unknowns

$$
H_{1}=\mathcal{D}_{1}\left(u_{2}\right)(0, .) \quad \text { and } \quad H_{2}=\mathcal{D}_{2}\left(u_{1}\right)(0, .)
$$

see [9].

At this point, it should be noted that the analysis of the present paper is restricted to rather idealistic geometries. However, the same formalism can be used for a domain decomposition into an arbitrary number of subdomains [12]. It has also been found there that the convergence estimates provided in this simple geometry predict very accurately the ones observed in practice even for complicated interface boundaries.

We first define interface conditions that lead to convergence in two steps of the algorithm. Let

$$
\Lambda_{i}=C_{i}^{1 / 2} A_{i}^{1 / 2} C_{i}^{1 / 2}
$$


where $A_{i}:=C_{i}^{-1 / 2} B_{i} C_{i}^{-1 / 2}$. Taking

$$
\mathcal{D}_{1}=\left(C_{1} \frac{\partial}{\partial n_{1}}+\Lambda_{2}\right) \quad \text { and } \quad \mathcal{D}_{2}=\left(C_{2} \frac{\partial}{\partial n_{2}}+\Lambda_{1}\right)
$$

leads to a convergence in two steps of (1), see [9]. This result is optimal in terms of iteration counts. But, matrices $\Lambda_{i}$ are a priori full matrices of order $n$ costly to compute and use. Instead, we will use approximations in terms of sparse matrices denoted $\Lambda_{i, a p}$. We lose convergence in two steps. In order to have the best convergence rate, we choose optimized sparse approximations to $\Lambda_{i}$ w.r.t the domain decomposition method.

We first consider diagonal approximations to $\Lambda_{i}$. At the continuous level, they correspond to Robin interface conditions. For a matrix $F$, let $\lambda_{m, M}(F)$ denote respectively the smallest and largest eigenvalues of $F$ and $\operatorname{diag}(F)$ the diagonal matrix made of the diagonal of $F$. We define

$$
\Lambda_{i, a p}^{0}=\tilde{\beta}_{i, o p t} \tilde{D}_{i}
$$

where $\tilde{D}_{i}:=C_{i}^{1 / 2} \operatorname{diag}\left(A_{i}\right)^{1 / 2} C_{i}^{1 / 2}$ and

$$
\tilde{\beta}_{i, o p t}=\sqrt{\beta_{m} \beta_{M}}
$$

with

$$
\beta_{m, M}=\sqrt{\lambda_{m, M}\left(\operatorname{diag}\left(A_{i}\right)^{-1 / 2} A_{i} \operatorname{diag}\left(A_{i}\right)^{-1 / 2}\right)}
$$

We also consider sparse approximations that will have the same sparsity as $A_{i}$. Let $\lambda_{m, M}=\lambda_{m, M}\left(\tilde{D}_{i}^{-2} A_{i}\right)^{1 / 2}$, the real parameters $\beta_{1}$ and $\beta_{2}$ are defined as follows

$$
\begin{gathered}
\beta_{1} \beta_{2}=\lambda_{m} \lambda_{M} \\
\beta_{1}+\beta_{2}=\left(2 \sqrt{\lambda_{m} \lambda_{M}}\left(\lambda_{m}+\lambda_{M}\right)\right)^{1 / 2}
\end{gathered}
$$

We define

$$
\Lambda_{a p, \beta_{1}, \beta_{2}}^{2}:=C_{i}^{1 / 2} \frac{\tilde{D}_{i}^{-1} A_{i}+\beta_{1} \beta_{2} \tilde{D}_{i}}{\beta_{1}+\beta_{2}} C_{i}^{1 / 2}
$$

At the continuous level, they correspond to optimized of order 2 interface conditions. The motivation for definitions (8) and (11) are given in [9].

\subsection{Numerical results}

The substructured problems are solved by a GMRES algorithm [29]. In the tables and figures, opt0 refers to (8) and opt2 to formula (11). In figure 4, we compare them with interface conditions obtained using a "frozen" coefficient approach. In the latter case, the interface conditions depend only locally on the coefficients of the problem, see [36] at the continuous level, [13] at the semi-discrete level and [28] at the algebraic level. We see a plateau in the 
convergence curve which can be related to a few very small eigenvalues in the spectrum of the substructured problem, see figure 4. A possible cure to this problem is the use of deflation methods, [21], [16], [22] and [30]. They rely on an accurate knowledge of the eigenvectors corresponding to the "bad" eigenvalues. With the opt2 interface conditions, no eigenvalue is close to zero and we need only extremal eigenvalues (and not the eigenvectors) of an auxiliary matrix. We also give comparisons with the Neumann-Neumann [33] [4] or FETI [18] approach, see figure 5. In the numerical tests, we have typically ten layers in each subdomain. In each layer, the diffusion tensor is anisotropic. We have jumps in the coefficients both across and along the interface. We are thus in a situation where the Neumann-Neumann or FETI methods are not necessarily optimal.

Fig. 4. Left: Convergence curve for various interface conditions. Right: Eigenvalues of the interface problem for opt2 (cross) and "frozen" (circles) interface conditions.
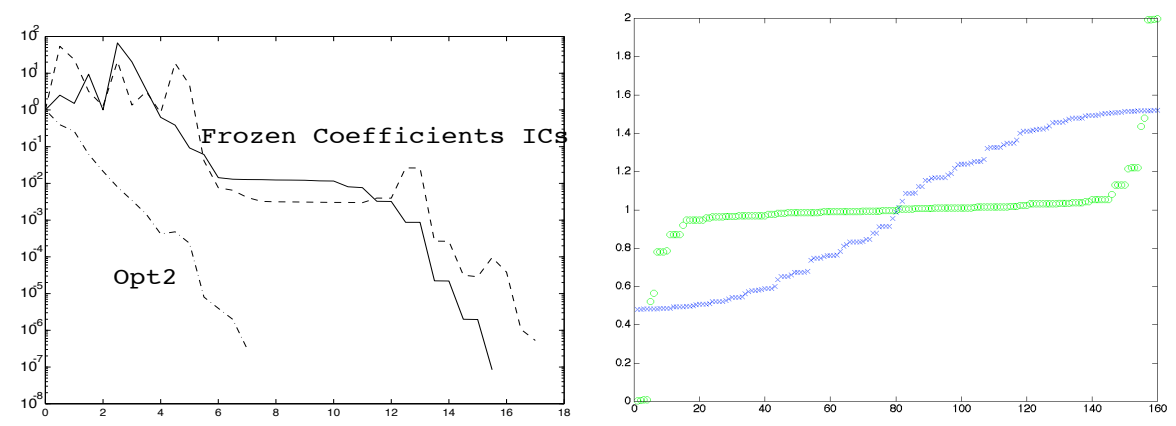

Fig. 5. residual vs. subdomain solve counts

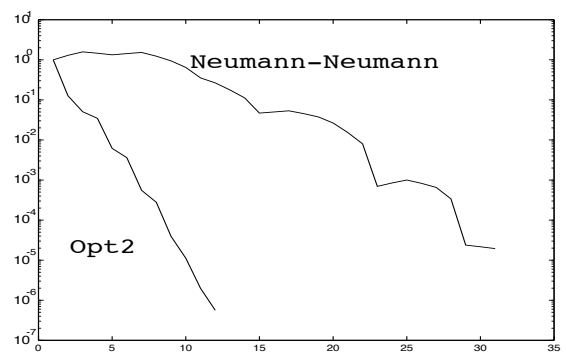




\section{Conclusion}

We have first recalled known results on optimized Schwarz methods for smooth coefficients operators. We have then considered problems with highly anisotropic and discontinuous coefficients, for which plateaus in the convergence of Krylov methods exist even when using "good" preconditioners. A classical remedy is to use deflated Krylov methods. We have developed in this paper a new algebraic approach in the DDM framework. We propose a way to compute optimized interface conditions for domain decomposition methods for symmetric positive definite equations. Compared to deflation, only two extreme eigenvalues have to be computed. Numerical results show that the approach is efficient and robust even with highly discontinuous coefficients both across and inside subdomains. The non-symmetric case is considered in this volume at the algebraic level in a joint work with Luca Gerardo-Giorda, see also [14]. The optimization of the interface condition is then much more difficult. Let us mention that such interface conditions can be used on non matching grids, see [1] and [7].

\section{References}

1. Yves Achdou, Caroline Japhet, Yvon Maday, and Frédéric Nataf. A new cement to glue non-conforming grids with Robin interface conditions: the finite volume case. Numer. Math., 92(4):593-620, 2002.

2. Jean-David Benamou and Bruno Deprés. A domain decomposition method for the helmholtz equation and related optimal control problems. J. of Comp. Physics, 136:68-82, 1997.

3. Jean-François Bourgat, Roland Glowinski, Patrick Le Tallec, and Marina Vidrascu. Variational formulation and algorithm for trace operator in domain decomposition calculations. In Tony Chan, Roland Glowinski, Jacques Périaux, and Olof Widlund, editors, Domain Decomposition Methods, pages 316, Philadelphia, PA, 1989. SIAM.

4. Lawrence C. Cowsar, Jan Mandel, and Mary F. Wheeler. Balancing domain decomposition for mixed finite elements. Math. Comp., 64(211):989-1015, July 1995.

5. Bruno Després. Décomposition de domaine et problème de Helmholtz. C.R. Acad. Sci. Paris, 1(6):313-316, 1990.

6. B. Engquist and A. Majda. Absorbing boundary conditions for the numerical simulation of waves. Math. Comp., 31(139):629-651, 1977.

7. I. Faille, F. Nataf, L. Saas, and F. Willien. Finite volume methods on nonmatching grids with arbitrary interface conditions and highly heterogeneous media. In Kornhuber R.; Hoppe R.; Périaux J.; Pironneau O.; Widlund O.; Xu J., editor, Domain Decomposition Methods in Science and Engineering Series, Lecture Notes in Computational Science and Engineering, Vol. 40, 2004.

8. C. Farhat and F. X. Roux. An unconventional domain decomposition method for an efficient parallel solution of large-scale finite element systems. SIAM J. Sc. Stat. Comput., 13:379-396, 1992. 
9. E. Flauraud and F. Nataf. Optimized interface conditions in domain decomposition methods. application at the semi-discrete and at the algebraic level to problems with extreme contrasts in the coefficients. Technical Report R.I. 524, CMAP, Ecole Polytechnique, 2004.

10. D. Funaro, A. Quarteroni, and P. Zanolli. An iterative procedure with interface relaxation for domain decomposition methods. SIAM J. Numer. Anal., 25(6):1213-1236, 1988.

11. Martin J. Gander and Gene H. Golub. A non-overlapping optimized Schwarz method which converges with an arbitrarily weak dependence on h. In Fourteenth International Conference on Domain Decomposition Methods, 2002.

12. Martin J. Gander, Frédéric Magoulès, and Frédéric Nataf. Optimized Schwarz methods without overlap for the Helmholtz equation. SIAM J. Sci. Comput., 24(1):38-60 (electronic), 2002.

13. Menno Genseberger. Domain decomposition in the Jacobi-Davidson method for eigenproblems. PhD thesis, Utrecht University, September 2001.

14. Luca Gerardo Giorda and Frédéric Nataf. Optimized schwarz methods for unsymmetric layered problems with strongly discontinuous and anisotropic coefficients. Technical Report 561, CMAP, CNRS UMR 7641, Ecole Polytechnique, France, 2004. submitted.

15. D. Givoli. Numerical methods for problems in infinite domains. Elsevier, 1992.

16. I.G. Graham and M.J. Hagger. Unstructured additive schwarz-cg method for elliptic problems with highly discontinuous coefficients. SIAM J. Sci. Comp., 20:2041-2066, 1999.

17. Thomas Hagstrom, R. P. Tewarson, and Aron Jazcilevich. Numerical experiments on a domain decomposition algorithm for nonlinear elliptic boundary value problems. Appl. Math. Lett., 1(3), 1988.

18. Axel Klawonn, Olof Widlund, and Maksymilian Dryja. Dual-primal FETI methods for three-dimensional elliptic problems with heterogeneous coefficients. Technical Report 815, Courant Institute of Mathematical Sciences, Department of Computer Science, April 2001. to appear in SIAM J. Numer. Anal.

19. Pierre-Louis Lions. On the Schwarz alternating method. III: a variant for nonoverlapping subdomains. In Tony F. Chan, Roland Glowinski, Jacques Périaux, and Olof Widlund, editors, Third International Symposium on Domain Decomposition Methods for Partial Differential Equations , held in Houston, Texas, March 20-22, 1989, Philadelphia, PA, 1990. SIAM.

20. Gert Lube, Lars Mueller, and Hannes Mueller. A new non-overlapping domain decomposition method for stabilized finite element methods applied to the nonstationary Navier-Stokes equations. Numer. Lin. Alg. Appl., 7:449-472, 2000.

21. Ronald B. Morgan. GMRES with deflated restarting. SIAM J. Sci. Comput., 24, 2002 .

22. R. Nabben and C. Vuik. A comparison of deflation and coarse grid correction applied to porous media flow. Technical Report R03-10, Delft University of Technology, 2003.

23. Frédéric Nataf. Interface connections in domain decomposition methods. In Modern methods in scientific computing and applications (Montréal, QC, 2001), volume 75 of NATO Sci. Ser. II Math. Phys. Chem., pages 323-364. Kluwer Acad. Publ., Dordrecht, 2002.

24. Fréderíc Nataf and Francois Rogier. Factorization of the convection-diffusion operator and the Schwarz algorithm. $M^{3} A S, 5(1): 67-93,1995$. 
25. Frédéric Nataf, Francois Rogier, and Eric de Sturler. Optimal interface conditions for domain decomposition methods. Technical Report 301, CMAP (Ecole Polytechnique), 1994.

26. Francis Nier. Remarques sur les algorithmes de décomposition de domaines. In Seminaire: Équations aux Dérivées Partielles, 1998-1999, pages Exp. No. IX, 26. École Polytech., 1999.

27. Alfio Quarteroni and Alberto Valli. Domain Decomposition Methods for Partial Differential Equations. Oxford Science Publications, 1999.

28. F.X. Roux, F. Magoulès, S. Salmon, and L. Series. Optimization of interface operator based on algebraic approach. In Ismael Herrera, David E. Keyes, Olof B. Widlund, and Robert Yates, editors, 14th International Conference on Domain Decomposition Methods, Cocoyoc, Mexico, 2002.

29. Y. Saad and M. H. Schultz. GMRES: A generalized minimal residual algorithm for solving nonsymmetric linear systems. SIAM J. Sci. Stat. Comp., 7:856-869, 1986.

30. Y. Saad, M. Yeung, J. Erhel, and F. Guyomarc'h. A deflated version of the conjugate gradient algorithm. SIAM J. Sci. Comput., 21(5):1909-1926 (electronic), 2000. Iterative methods for solving systems of algebraic equations (Copper Mountain, CO, 1998).

31. H. A. Schwarz. Über einen Grenzübergang durch alternierendes Verfahren. Vierteljahrsschrift der Naturforschenden Gesellschaft in Zürich, 15:272-286, May 1870.

32. Barry F. Smith, Petter E. Bjørstad, and William Gropp. Domain Decomposition: Parallel Multilevel Methods for Elliptic Partial Differential Equations. Cambridge University Press, 1996.

33. Patrick Le Tallec and Marina Vidrascu. Generalized Neumann-Neumann preconditioners for iterative substructuring. In Petter E. Bjørstad, Magne Espedal, and David Keyes, editors, Domain Decomposition Methods in Sciences and Engineering. John Wiley \& Sons, 1997. Proceedings from the Ninth International Conference, June 1996, Bergen, Norway.

34. Kian H. Tan and Mart J. A. Borsboom. On generalized Schwarz coupling applied to advection-dominated problems. In David E. Keyes and Jinchao Xu, editors, Seventh International Conference of Domain Decomposition Methods in Scientific and Engineering Computing, pages 125-130. AMS, 1994. Held at Penn State University, October 27-30, 1993.

35. Andrea Toselli and Olof Widlund. Domain Decomposition Methods - Algorithms and Theory, volume 34 of Springer Series in Computational Mathematics. Springer, 2004.

36. Françoise Willien, Isabelle Faille, Frédéric Nataf, and Frédéric Schneider. Domain decomposition methods for fluid flow in porous medium. In 6th European Conference on the Mathematics of Oil Recovery, September 1998.

37. Barbara Wohlmuth. Discretization Methods and Iterative Solvers Based on Domain Decomposition, volume 17 of Lecture Notes in Computational Science and Engineering. Springer, 2001. 